\title{
Downregulation of Cardiotrophin-Like Cytokine 1(CLCF1) Inhibits Osteoblastic Differentiation by Regulating RANKL/ OPG Pathway
}

\section{Juan Chen}

Fujian Medical University https://orcid.org/0000-0001-5639-7615

Huijuan Xu

Fujian Academy of Agricultural Sciences Institute of Plant Protection

\section{Yunjing Ye}

Fujian Medical University

\section{Lihua Xie}

Fujian Medical University

\section{Shengqiang Li}

Fujian Medical University

Jirong Ge ( $\nabla$ gjrrjgcy@sina.com )

Fujian Medical University

\section{Research article}

Keywords: cardiotrophin-like cytokine factor 1, osteoprotegerin, cytokine receptor activator of the nuclear factor-kB ligand, JAK2/STAT3 signal pathway, postmenopausal osteoporosis

Posted Date: November 20th, 2020

DOl: https://doi.org/10.21203/rs.3.rs-111138/v1

License: (9) This work is licensed under a Creative Commons Attribution 4.0 International License. Read Full License 


\section{Downregulation of Cardiotrophin-like cytokine 1(CLCF1) Inhibits osteoblastic}

differentiation by regulating RANKL/ OPG Pathway

Juan Chen $\uparrow$, Huijuan $\mathrm{Xu} \uparrow$, Yunjing Ye, Lihua Xie, Shengqiang Li and Jirong Ge *

* Correspondence: gjrrjgcy@sina.com

$\dagger$ Juan Chen and Huijuan Xu contributed equally to this work.

Fujian Academy of Chinese Medicine Sciences, Fuzhou 350001, People's Republic of China 


\begin{abstract}
Background: Cardiotrophin-like cytokine factor 1 (CLCF1) is a member of the IL (interleukin)-6-type cytokine. Although its immunomodulatory functions are well defined, data on the physiological and pathological functions of the CLCF1 in bone metabolism remains scant. Here, we interrogated the functions and mechanisms of CLCF1 in osteoblast differentiation.
\end{abstract}

Methods: A total of 109 patients with postmenopausal osteoporosis (PMOP) and 94 control group participants were included in this study. Quantitative reverse transcription PCR (RT-qPCR) and Western blot techniques were used to profile the expression of the CLCF1, nuclear factor-kB ligand and its decoy receptor osteoprotegerin (RANKL/OPG), as well as the janus activated kinase (JAK2)/transcription3 (STAT3) pathway. To elucidate the effect of CLCF1 on bone metabolism, we established CLCF1 geneknockout Raji cell lines and Raji-MG-63 co-culture system, and interrogated the osteoblast differentiation by measuring the activity of ALP and the level of Alizarin-Red S staining.

Results: Our data demonstrated that the expression of CLCF1 was highly downregulated in PMOP compared with the control group. Moreover, the level of CLCF1 was proportional to the bone mineral density (BMD) in the postmenopausal osteoporosis. Furthermore, the deletion of CLCF1 gene inhibits osteoblast differentiation by regulating the RANKL/OPG system.

Conclusion: Our findings unravel the previously unidentified roles of CLCF1 in the bone-immune crosstalk, as well as potential therapeutic strategies for postmenopausal osteoporosis. 
Keywords: cardiotrophin-like cytokine factor 1, osteoprotegerin, cytokine receptor activator of the nuclear factor-kB ligand, JAK2/STAT3 signal pathway, postmenopausal osteoporosis

\section{Introduction}

Postmenopausal osteoporosis (PMOP) is a chronic systemic skeletal disorder characterized by bone loss and bone fragility. Although PMOP is traditionally viewed as an endocrine disease, recent studies have shown that PMOP might be a chronic inflammatory bone disease [1-3]. Most osteoimmunology findings have robustly linked the bone loss phenomenon with the immune system [4]. It is now widely recognized that the immune cells and cytokine effectors contribute to bone loss. For example, tumor necrosis factor- $\alpha$ (TNF- $\alpha$ ), interleukin-1 (IL-1) and IL-6 have a similar impact in favor of bone resorption $[5,6]$. In addition, nuclear factor-kB ligand (RANKL) and its decoy receptor osteoprotegerin (OPG) have been shown to be a key determinant cytokine for osteoclastogenesis [7].

Cardiotrophin-like cytokine factor 1(CLCF1), also known as the neurotrophin-1 (NNT1) or B-cell stimulatory factor-3 (BSF-3), has been shown to mediate important functions in the survival and development of motor neurons [8,9]. It can be efficiently secreted together with its heteromeric partners such as soluble ciliary neurotrophic factor receptor (sCNTFR) and cytokine receptor-like factor-1 (CRLF-1). A more recent study has postulated the physiological and pathological functions of CLCF1 in a wide range of organ systems. Notably, as a member of the IL-6 cytokine family, CLCF1 exerts immunomodulatory functions, such as supporting B-cell expansion and humoral biology 
[10,11]. Besides, IL-6 family cytokines such as IL-6, ciliary neurotrophic factor (CNTF), leukemia inhibitory factor (LIF) and cardiotrophin-1 (CT-1) also contribute to the homeostasis of bone $[12,13]$. However, the role of the CLCF1 in the interaction between the immune system and bone biology is yet to be defined.

Here, we demonstrate that the expression of circulating CLCF1 is downregulated in patients with PMOP. Moreover, the CLCF1 expression is positively correlated with BMD and circulating lymphocyte counts. On the other hand, deletion of CLCF1 affects the RANKL/OPG ratio through regulation of the Janus activated kinase (JAK2)/transcription 3 (STAT3) pathway. Taken together, our findings define a novel role for CLCF1 in the osteoimmunology effect and give more insights into the pathogenesis of PMOP.

\section{Material and Methods}

\section{Participants and study design}

Study participants were recruited from the outpatient department of the Fujian Academy of Chinese Medicine Sciences, Fuzhou, P.R. China between January 2016 and May 2018. A total of 203 women (94 healthy controls and 109 PMOP patients) were recruited in this study. We included 55-75 years old women with at least 1 year after menopause. OP was defined as BMD T-score $\leq-2.5 \mathrm{SD}$ at the femoral neck and/or lumbar spine according to WHO criteria. The PMOP group included women with at least one OP, and with clinical peripheral or vertebral fracture confirmed by X-ray. On the other hand, the healthy control group included women with no history of fracture and with normal BMD. We excluded women on previous or current anti-OP treatment, treatment that would interfere with the immune system, those with Rheumatoid arthritis, diabetes mellitus, 
hyperthyroidism, and other secondary osteoporosis. The current study was reviewed and approved by the Committee of the Fujian Academy of Chinese Medicine Sciences and was performed in line with the declaration of Helsinki. All the participants gave an informed written consent.

\section{Cell culture}

Raji cells (cat. no. TCHu 44) and MG-63 cells (cat. no. TCHu 124) were obtained from the Stem Cell Bank, Chinese Academy of Sciences. The cells were then cultured in RPMI-1640 medium (Gibco, Grand Island), supplemented with 10\% fetal bovine serum (FBS, Gibco, Grand Island), and $50 \mathrm{U} / \mathrm{ml}$ penicillin/streptomycin (Gibco, Grand Island) at $37{ }^{\circ} \mathrm{C}$, and a humidified atmosphere of $5 \% \mathrm{CO}_{2}$. Recombinant human CLCF1 (rhCLCF1) was obtained from Abcam, MA, USA, while the JAK2 inhibitor AG490 was purchased from Selleck Chemicals, Houston, TX. The Raji cells were treated with recombinant CLCF1 (5 ng/mL), AG490 (10 $\mu \mathrm{g} / \mathrm{mL})$, and a combination of CLCF1 and AG490 for 1 hour.

\section{Bone mineral density (BMD) and biological measurements}

All the participants underwent the lumbar spine and left hip BMD evaluation by dualenergy X-ray absorptiometry (Hologic Discovery W Bone Densitometer, USA). We then approximated the white blood cells and lymphocyte count from an aliquot of blood sample using an automated analyzer (Sysmex XE5000, Sysmex Corporation, Kobe, Japan).

\section{Generation of CLCF1 gene deficient cell lines}


Lenti-mediated CLCF1 gene-knockout cell lines were generated with the CRISPR/ Cas9 system as previously reported[14]. CLCF1 sgRNA sequence 5'-TTGAAGTCT GGCTCGTTGAA-3' was cloned into the GV371 vector (Genechem, Shanghai, China). Raji cells were then transfected at a multiplicity of infection (MOI) of 100 with the lentivirus vector, and cultured in puromycin $(4 \mu \mathrm{g} / \mathrm{ml})$ pressure. Analysis of the gene deletion was confirmed by Western blot analysis. Unless stated otherwise, each group of experiments was repeated three times.

\section{Establishment of the Raji-MG-63 co-culture system and Osteogenic differentiation}

A co-culture system was established using transwell experiments. Briefly, the Raji cells were seeded on a 24-well plate, with cell line MG-63 making the lower layer. The cells were cultured in complete medium (RPMI1640 medium $+10 \%$ fetal bovine serum $+1 \%$ double-antibody). The MG-63 cells were subjected to osteogenic differentiation as reported previously [15], and the osteogenesis efficiency was assessed by the ALP activity and calcium deposition (Alizarin Red S staining).

\section{Reverse transcription-quantitative PCR (RT-qPCR)}

Peripheral blood mononuclear cells (PBMCs) were isolated from the control group and PMOP patient samples using Ficoll density gradient. Total RNA was extracted using the TRIzol reagent (Invitrogen, CA, USA), and then $1 \mu \mathrm{g}$ of the total RNA was used for reverse transcription. The gene expression profile was analyzed by RT-qPCR assays performed on the ABI 7500 Fast platform (Applied Biosystems; Foster City, CA, USA). The primer pair sequences were: CLCF1 forward, 5'- CCTCCTGCTTCTT GCC-TACC3'and reverse,5'-TGCCACCATGTTTGTTTCCT -3'. ACTIN forward, 5'-GGGAAACT 
GTGGCGTGAT-3'and reverse, 5'-GAGTGGG TGTCGCTGTT-GA-3'. Three replicate wells were performed for each sample, normalized by internal reference ACTIN, and then the relative quantitative analysis was done using the $2-{ }^{\Delta \Delta \mathrm{Cq}}$ method [16]. All the experiments were conducted in triplicates.

\section{Western blot}

The PBMCs were confirmed by western blot analysis and separated by Ficoll density gradient. Total cellular protein was isolated using the RIPA buffer (Beyotime, Jiangsu, China). The extracted proteins were resolved in $10 \%$ sodium dodecyl sulfatepolyacrylamide electrophoresis (SDS-PAGE) gels and transferred onto the PVDF membrane. Antibodies against CLCF1 (cat.no. ab251886; 1:1,000), OPG (cat.no. ab73400; 1:1,000), RANKL (cat.no. ab9957; 1:1,000) (all from Abcam, MA, USA) and JAK2 (cat.no.3230; 1:1,000), p-JAK2 (cat.no. 3771; 1:1,000), STAT3 (cat.no. 12640; 1:1,000), p-STAT3 (cat.no. 52075; 1:1,000), ACTIN (cat.no. 3700; 1:1,000) (all from CST, MA, USA) were used for the development of the blots. Image-Pro Plus software (NIH, USA) was used for Semi-quantitative analysis of the protein bands' gray value. All the experiments were performed in triplicates.

\section{Statistical analysis}

SPSS 18.0 software (SPSS Inc., Chicago, IL, USA) was used for statistical analysis and the data were described by mean values with standard deviations (SDs). Non-parametric tests Kruskal-Wallis, and Mann-Whitney were used to compare the differencse between the two groups. Spearman's correlation was performed to profile the relationships 
between CLCF1 expression and clinical variables. A $P<0.05$ was considered to be statistically significant.

\section{Results}

\section{Demographic and clinical characteristics of the participants}

A total of 109 patients with PMOP and 94 control group participants were included in this study. The demographics and clinical characteristics of the study population are summarized in Table 1. Age at puberty and menopause did not significantly differ in the two groups. Whereas the PMOP patients had lower weight and height, there was no significant difference in BMI for the PMOP group $(24.03 \pm 2.89 \mathrm{~kg} / \mathrm{m} 2)$ as compared with the healthy control group $(23.94 \pm 3.33 \mathrm{~kg} / \mathrm{m} 2)$. Interestingly, there were significantly upregulated white blood cells $(p=0.009)$, red blood cells $(p=0.007)$, platelets $(p=$ 0.012), and hemoglobin ( $p=0.005)$ in the PMOP patients (Table 1).

Table 1 Clinical characteristics of the study participants

\begin{tabular}{lccc}
\hline Variables & Control $(\mathrm{n}=94)$ & $\mathrm{PMOP}(\mathrm{n}=109)$ & $\mathrm{P}$ value \\
\hline Age $($ years $)$ & $61.50 \pm 5.51$ & $62.20 \pm 5.76$ & 0.377 \\
Age of menopause (year) & $49.52 \pm 4.73$ & $50.04 \pm 5.41$ & 0.468 \\
Age of puberty (year) & $12.86 \pm 1.23$ & $13.03 \pm 1.34$ & 0.348 \\
Height $(\mathrm{cm})$ & $157.23 \pm 3.13$ & $155.84 \pm 4.23$ & 0.009 \\
Weight $(\mathrm{kg})$ & $58.90 \pm 8.98$ & $56.24 \pm 8.12$ & 0.027 \\
$\mathrm{BMI}\left(\mathrm{kg} / \mathrm{m}^{2}\right)$ & $23.94 \pm 3.33$ & $24.03 \pm 2.89$ & 0.836 \\
WBC $\left(\times 10^{9} / \mathrm{L}\right)$ & $5.56 \pm 1.34$ & $5.09 \pm 1.23$ & 0.009 \\
$\mathrm{RBC}\left(\times 10^{12} / \mathrm{L}\right)$ & $4.03 \pm 0.45$ & $4.18 \pm 0.34$ & 0.007 \\
$\mathrm{HGB}(\mathrm{g} / \mathrm{L})$ & $123.32 \pm 12.23$ & $127.45 \pm 11.34$ & 0.012 \\
$\mathrm{PLT}\left(\times 10^{9} / \mathrm{L}\right)$ & $196.45 \pm 43.76$ & $215.43 \pm 51.78$ & 0.005 \\
\hline
\end{tabular}

Notes: Data expressed as mean \pm standard deviation (SD), $P>0.05$ was considered as nonsignificant.

Abbreviations: BMI, body mass index; WBC, white blood cell; RBC= red blood cell, HGB, hemoglobin; PLT, platelets. 


\section{CLCF1 expression is downregulated in patients with PMOP}

We evaluated the expression of CLCF1 in the peripheral blood of the participants by RT-qPCR and Western blot analysis. Compared with the healthy individuals, our data showed a significantly reduced CLCF1 mRNA expression in PMOP patients $(p<0.001$,

Fig. 1A). The results were validated by western blot analysis which showed a significant downregulation of the CLCF1 protein in the circulating blood of the PMOP patients compared with that of healthy controls $(p<0.001$, Fig. 1B). These data demonstrates that CLCF1 is indeed dysregulated in PMOP.

\section{The expression of CLCF1 in the circulating blood correlates with BMD and}

\section{lymphocytes}

To better understand the observed CLCF1 expression changes in PMOP patients, we explored the correlation between the blood circulation CLCF1 and BMD as well as peripheral blood cell count (Fig. 2). Here, the data demonstrated that the expression of CLCF1 was positively correlated with lumbar BMD $(r=0.368, p<0.001$, Figure 2A) and femoral neck BMD $(r=0.382, p<0.001$, Fig. 2B). Besides, the CLCF1 expression was markedly related to the intensity of peripheral blood lymphocytes $(r=0.489, p<$ 0.001, Fig. 2C). However, there was no significant correlation between the CLCF1 levels and white blood cell counts $(\mathrm{r}=0.162, p<0.001$, Fig. 2D). Our results demonstrated that CLCF1 expression profile might serve as a candidate marker of PMOP.

\section{Dysregulated expression of CLCF1 affects RANKL/ OPG via the JAK2/STAT3 pathway}


CLCF1 is a known B cell-stimulating factor and has shown its capacity to stimulate B cell expansion. On the other hand, B cells are a considered as a source of OPG and the RANKL. To determine whether the CLCF1 gene affects bone metabolism via the regulation of B cells, we generated CLCF1 deficient Raji cells. As expected, deletion of CLCF1 significantly reduced OPG expression but marginally affected RANKL expression (Fig. 3B, C). The knockout of CLCF1 gene increased the RANKL/OPG ratio compared to the control group (Fig. 3D).

To further assess the function of CLCF1 in the modulation of the JAK2/STAT3 pathway, we incubated Raji cells with the JAK2 inhibitor AG490 before the addition of recombinant CLCF1 (rhCLCF1). Our data demonstrated that the CLCF1 robustly increased the JAK2 phosphorylation, and the inhibitor AG490 blocked the rhCLCF1induced JAK2/STAT3 activation (Fig 3A). Besides, the CLCF1 gene deletion diminished the p-JAK2/JAK2 and p-STAT3/STAT3 ratio (Fig. 3B, C). Collectively, our findings highlighted the crucial role played by the CLCF1 gene in the modification of the RANKL/OPG ratio via the JAK2/STAT3 pathway.

\section{Deletion of the CLCF1 gene suppresses osteoblast differentiation in the Raji-MG-63 co-culture system}

To define whether the CLCF1 gene affects osteoblast differentiation via the RANKL/OPG system, we established a Raji-MG-63 co-culture system. We assayed the osteoblast differentiation by measuring the activity of ALP and the level of Alizarin Red S staining. Our results showed that in the CLCF1 deficient Raji cells, there is a compromised osteoblast differentiation ability in the MG-63 (Fig. 4B, C). Similarly, and 
in consistent with the reduced expression of CLCF1, the RANKL/OPG ration increased (Fig. 4A).

\section{Discussion}

As a cytokine of the IL-6 family, CLCF1 was earlier associated with the regulation of B cell expansion and motor neuron development. A more recent study established potential activities in a wide range of biology and degenerative conditions[19]. However, the functions of CLCF1 in the physiology and pathology of bone metabolism is yet to be defined. Here, we demonstrated that CLCF1 in blood circulation was downregulated in the PMOP patients. Furthermore, the CLCF1 expression profile was positively correlated with BMD and circulating lymphocyte counts. Besides, deletion of CLCF1 significantly inactivated the JAK2/ STAT3 pathway and increased the RANKL/OPG ratio. The CLCF1 gene knock out inhibited osteoblast differentiation. Taken together, our findings demonstrate that CLCF1 may play a vital role in immune-mediated bone loss by modulating the RANKL/OPG balance.

Accumulating evidence has revealed the close cross-talk underlying the relationship between the bone and immune systems. A large number of immune cells affect bone resorption and formation [20,21]. The concept of osteoimmunology pointed out that chronic inflammatory diseases such as osteoporosis, rheumatoid arthritis, and periodontitis, induce bone loss through the stimulation of cytokines synthesized by activated immune cells [22]. In this respect, many cytokines that are classically considered osteoimmune-related, such as Inter Leukins 6 (IL-6) [23], tumor necrosis factor-alpha (TNF- $\alpha$ ) [24], macrophage colony-stimulating factor (M-CSF) [25], 
Receptor-Activator of Nuclear factor Kappa B (RANK), and its ligand (RANKL) [26], have all been found to define the biology and physiology of the bone. CLCF1 has been shown to be highly expressed in adult immune cells. As a member of the IL-6 cytokine family, the main roles of CLCF1 were thought to be restricted to support motor neuron survival and development $[17,18]$.

Recent studies have highlighted possible CLCF1 roles in immuno-modulatory functions and B-cell expansion. Members of the IL-6 family, such as ciliary neurotrophic factor (CNTF), leukemia inhibitory factor (LIF), cardiotrophin-1 (CT-1), and oncostatin M (OSM) have been shown to stimulate differentiation of osteoblasts and promote bone resorption in pathological conditions. Unlike the other IL-6 family cytokines, data on the role of CLCF1 in OP remains scant [27,28]. In our study, we showed that CLCF1 is downregulated in PMOP patients, and the phenotype correlated with low BMD as well as decreased lymphocyte count in blood. Further study of the relationship between CLCF1 and bone metabolism and their crosstalk may have important clinical implications.

Bone homeostasis is regulated by a balance between bone formation and resorption, and any disruption of the balance results in bone disease. Most importantly, the intersection of the OPG/RANK/ RANKL pathway in bone biology and immunity has been extensively reported $[29,30]$. RANKL is responsible for the stimulation of osteoclast differentiation and bone resorption while OPG interdicts bone resorption by blocking RANKL. Therefore, it is feasible to evaluate the expression and the relative ratio of RANKL/OPG in the pathogenesis of osteodestructive processes.

Furthermore, emerging evidence illustrates that B cells are sources of RANKL or OPG, and play a vital role in the biology of the bone [31]. Our study showed that the deletion of 
CLCF1 gene reduced OPG expression, and leads to the dysregulation of the RANKL/OPG ratio. As anticipated, the CLCF1 expression affected the survival and expansion of the B cell population [32]. Thus, the CLCFI gene might be exerting a crucial influence in bone loss via the regulation of the RANKL/OPG pathway in B cells. This finding differs from previous studies which showed that CLCF1 prevents osteoblast generation and mineralization in mouse MSCs [33]. We presume that the discrepancies may be due to the different cell lines and study design, and requires confirmation by further studies.

Mechanistically, our findings demonstrated that CLCF1 controls RANKL/OPG expression through the activation of the JAK2 /STAT3 pathway. In their study, Mukut Sharma et al [34] showed that CLCF1- induced up-regulation of STAT3 phosphorylation was eased up by the heterodimer CLCF1-CRLF1. On the other hand, Virginia J. Savinet et al.[35] reported that CLCF1 is a potential "circulating factor" in the human renal disease focal segmental glomerulosclerosis (FSGS), and it acts by initiating the JAK/STAT signaling pathway in glomerular podocytes. Besides, the JAK2/STAT3 pathway has been associated with a vital role in the origination and evolution of osteoporosis [36,37]. In particular, a recent study demonstrated that the JAK2/STAT3 pathway modulates the level of RANKL and enhances osteoclast. These findings do not only offer mechanistic insights on how CLCF1 influences RANKL/OPG axis, but also indicate cross-talk between CLCF1 and JAK2/STAT3 pathway.

\section{Conclusion}

Overall, our data demonstrated that CLCF1 levels are diminished in PMOP, and are positively correlated with BMD. We showed that CLCF1 promotes osteoporosis by 
targeting RANKL/OPG axis and regulating the JAK/STAT pathway. These results provide a novel understanding of the fundamental mechanism of immuno-skeletal interactions, and offer perspectives on CLCF1 as an innovative therapeutic target for pathological bone loss.

\begin{abstract}
Abbreviations
CLCF1 : Cardiotrophin-like cytokine factor 1 ; PMOP : postmenopausal osteoporosis ; RT-qPCR : Quantitative reverse transcription PCR ; RANKL : nuclear factor-kB ligand; OPG : osteoprotegerin; JAK2 : Janus activated kinase ; STAT3 :Transcription3 ; BMD : bone mineral density ; CRLF-1: cytokine receptor-like factor-1; sCNTFR: soluble ciliary neurotrophic factor receptor; LIF: leukemia inhibitory factor; CT-1: cardiotrophin-1
\end{abstract}

\title{
Acknowledgments
}

Not applicable.

\section{Authors' contributions}

GJR and XHJ conceived and designed the study; CJ,XLH and YYJ performed the experiment and collected the data. LSQ analyzed the data. CJ drafted the manuscript. LSQ and GJR contributed to review and editing of the article. All authors read and approved the final manuscript.

\section{Funding}

This study was supported by the Natural Science Foundation of Fujian Province, China (grant no. 2017J01535), and the National Natural Science Foundation of China (grant no. 81674007).

\section{Availability of data and materials}


The data from this study are available in this published article.

\section{Ethics approval and consent to participate}

This study was performed in accordance with the Declaration of Helsinki and with the approval of the Ethical Committee of the the Fujian Academy of Chinese Medicine

Sciences(No. 2016KY020-02; Approve date: August 11, 2016).

\section{Consent for publication}

Not applicable.

\section{Competing interest}

The authors declare no conflict of interest.

\section{References}

1. Rachner TD, Khosla S, Hofbauer LC. Osteoporosis: Now and the future. Lancet. 2011; 377 (9773):1276-87.

2. Graves DT, Li J, Cochran DL. Inflammation and uncoupling as mechanisms of periodontal bone loss. JDent Res. 2011;90(2):143-53.

3. Oliveira MC, Vullings J, van de Loo F. Osteoporosis and osteoarthritis are two sides of the same coin paid for obesity. Nutrition. 2020; 70:110486.

4. Ponzetti M, Rucci N. Updates on Osteoimmunology: What's New on the Cross-Talk Between Bone and Immune System. Front Endocrinol (Lausanne). 2019;10:236.

5. de Vries TJ, El BI, Kamradt T, Schett G, Jansen I, D'Amelio P. What are the peripheral blood determinants for increased osteoclast formation in the various inflammatory diseases associated with bone loss? Front Immunol. 2019;10:505.

6. Srivastava RK, Dar HY, Mishra PK. Immunoporosis: Immunology of OsteoporosisRole of t cells. Front Immunol. 2018;9:657.

7. Sobacchi C, Menale C, Villa A. The RANKL-RANK axis: A bone to thymus round trip. Front Immunol. 2019;10:629.

8. Lelievre E, Plun-Favreau H, Chevalier S, Froger J, Guillet C, Elson G C,et al. 
Signaling pathways recruited by the cardiotrophin-like cytokine/cytokine-like factor-1 composite cytokine: Specific requirement of the membrane-bound form of ciliary neurotrophic factor receptor alpha component. J Biol Chem. 2001; 276: 22476-84.

9. Larsen JV, Kristensen AM, Pallesen LT, Bauer J, Vægter CB, Nielsen MS, et al. Cytokine-Like Factor 1, an Essential Facilitator of Cardiotrophin-Like Cytokine: Ciliary Neurotrophic Factor Receptor alpha Signaling and sorLA-Mediated Turnover. Mol Cell Biol. 2016;36:1272-86.

10. Pasquin S, Tormo A, Moreau J, Laplante V, Sharma M, Gauchat JF, et al. Cardiotrophin-Like Cytokine Factor 1 Exhibits a Myeloid-Biased HematopoieticStimulating Function. Front Immunol. 2019;10:2133.

11. Senaldi G, Stolina M, Guo J, Faggioni R, McCabe S, Kaufman SA, et al. Regulatory effects of novel neurotrophin-1/b cell-stimulating factor-3 (cardiotrophin -like cytokine) on B cell function. J Immunol. 2002;168:5690-8.

12. Sims NA. Cell-specific paracrine actions of IL-6 family cytokines from bone, marrow and muscle that control bone formation and resorption. Int J Biochem Cell Biol. 2016;79:14-23.

13. Askmyr M, White KE, Jovic T, King HA, Quach JM, Maluenda AC, et al. Ciliary neurotrophic factor has intrinsic and extrinsic roles in regulating $\mathrm{B}$ cell differentiation and bone structure. Sci Rep. 2015;5:15529.

14. Hochheiser K, Kueh AJ, Gebhardt T, Herold MJ. CRISPR/Cas9: A tool for immunological research. Eur J Immunol. 2018;48(4):576-583.

15. Fan JZ, Yang X, Bi ZG. The effects of 6-gingerol on proliferation, differentiation, and maturation of osteoblast-like MG-63 cells. Braz J Med Biol Res. 2015; 48 (7):637-43.

16. Livak KJ, Schmittgen TD. Analysis of relative gene expression data using real-time quantitative PCR and the 2(-Delta Delta C(T)) Method. Methods. 2001;25(4):402-8.

17. Rose-John S. Interleukin-6 family cytokines. Cold Spring Harb Perspect Biol. 2018;10(2).

18. Jones SA, Jenkins BJ. Recent insights into targeting the IL-6 cytokine family in inflammatory diseases and cancer. Nat Rev Immunol. 2018;18(12):773-789. 
19. Sims NA. Cardiotrophin-like cytokine factor 1 (CLCF1) and neuropoietin (NP) signalling and their roles in development, adulthood, cancer and degenerative disorders. Cytokine Growth Factor Rev. 2015;26(5):517-22.

20. Nakamura MC. Osteoimmunology: Entwined regulation of integrated systems. Semin Immunopathol. 2019;41(5):547-549.

21. Srivastava RK, Dar HY, Mishra PK. Immunoporosis: Immunology of OsteoporosisRole of $\mathrm{t}$ cells. Front Immunol. 2018;9:657.

22. Terashima A, Takayanagi H. Overview of osteoimmunology. Calcif Tissue Int. 2018;102(5):503-511.

23. Wang T, He C. TNF-alpha and IL-6: The link between immune and bone system. Curr Drug Targets. 2020;21(3):213-227.

24. Ilesanmi-Oyelere BL, Schollum L, Kuhn-Sherlock B, McConnell M, Mros S, Coad $\mathbf{J}$, et al. Inflammatory markers and bone health in postmenopausal women: A crosssectional overview. Immun Ageing. 2019;16:15.

25. Zur Y, Rosenfeld L, Bakhman A, Ilic S, Hayun H, Shahar A, et al. Engineering a monomeric variant of macrophage colony-stimulating factor (M-CSF) that antagonizes the c-FMS receptor. Biochem J. 2017;474:2601-2617.

26. Liu Y, Wang C, Wang G, Sun Y, Deng Z,Chen L, et al. Loureirin B suppresses RANKL-induced osteoclastogenesis and ovariectomized osteoporosis via attenuating NFATc1 and ROS activities. Theranostics. 2019;9:4648-4662.

27. Sims NA, Walsh NC. GP130 cytokines and bone remodelling in health and disease. Bmb Rep. 2010;43:513-523.

28. McGregor NE, Poulton IJ, Walker EC, Pompolo S, Quinn JMW, Martin TJ, et al. Ciliary neurotrophic factor inhibits bone formation and plays a sex-specific role in bone growth and remodeling. Calcif Tissue Int. 2010; 86: 261-270.

29. Stuss M, Rieske P, Ceglowska A, Stêpień-Kłos W, Liberski PP, Brzeziańska E, et al. Assessment of OPG/RANK/RANKL gene expression levels in peripheral blood mononuclear cells (PBMC) after treatment with strontium ranelate and ibandronate in patients with postmenopausal osteoporosis. J Clin Endocrinol Metab. 2013;98:E1007-11.

30. Zhao J, Huang M, Zhang X, Xu J, Hu G, Zhao X, et al. MiR-146a deletion protects 
from bone loss in OVX mice by suppressing RANKL/OPG and M-CSF in bone microenvironment. J Bone Miner Res. 2019;34:2149-2161.

31. Xu S, Zhang Y, Liu B, Huang B, Yan B, Zhang Z, et al. Activation of mTORC1 in b lymphocytes promotes osteoclast formation via regulation of beta-Catenin and RANKL/OPG. J Bone Miner Res. 2016;31:1320-33.

32. Ge JR, Xie LH, Chen J, Li SQ, Xu HJ, Lai YL, et al. Liuwei Dihuang Pill () Treats Postmenopausal Osteoporosis with Shen (Kidney) Yin Deficiency via Janus Kinase/Signal Transducer and Activator of Transcription Signal Pathway by Upregulating Cardiotrophin-Like Cytokine Factor 1 Expression. Chin J Integr Med. 2018;24:415-422.

33. Nahlé S, Pasquin S, Laplante V, Rousseau F, Sharma M, Gauchat JF. Cardiotrophinlike Cytokine (CLCF1) Modulates Mesenchymal Stem Cell Osteoblastic Differentiation. J Biol Chem. 2019;294:11952-11959.

34. Sharma M, Zhou J, Gauchat JF, Sharma R, McCarthy ET, Srivastava T, et al. Janus kinase 2/signal transducer and activator of transcription 3 inhibitors attenuate the effect of cardiotrophin-like cytokine factor 1 and human focal segmental glomerulosclerosis serum on glomerular filtration barrier. Transl Res. 2015;166:38498.

35. Savin VJ, Sharma M, Zhou J, Gennochi D, Fields T, Sharma R, et al. Renal and hematological effects of CLCF-1, a B-Cell-Stimulating cytokine of the IL-6 family. J Immunol Res. 2015;2015:714964.

36. Li C, Zhao J, Sun L, Yao Z, Liu R, Huang J, et al. RANKL downregulates cell surface CXCR6 expression through JAK2/STAT3 signaling pathway during osteoclastogenesis. Biochem Biophys Res Commun. 2012;429:156-62.

37. Li CH, Xu LL, Jian LL, Yu RH, Zhao JX, Sun L, et al. Stattic inhibits RANKLmediated osteoclastogenesis by suppressing activation of STAT3 and NF-kappaB pathways. Int Immunopharmacol. 2018;58:136-144. 


\section{Figure legends:}

Fig. 1. Altered expression of CLCF1 in postmenopausal osteoporosis patients. (A) The relative CLCF1 mRNA expression as detected by RT-qPCR. (B) Western blot analysis of the quantitative expression of the relative CLCF1 protein. The data represent the mean expression values normalized to the housekeeping gene Actin. Values are means \pm SD of three independent experiments, ${ }^{*} \mathrm{P}<0.001$ compared to the $\mathrm{Ctrl}$ group.

Fig.2. Association of CLCF1 expression profile with bone mineral density (BMD) and peripheral blood cell counts in postmenopausal osteoporosis patients. (A) The relationship between the CLCF1 protein expression in peripheral blood cells and the BMD of the lumbar spine. (B) The relationship between the CLCF1 protein expression and femoral neck BMD. (C) Association of CLCF1protein expression with peripheral blood white blood cell counts. (D) Association of CLCF1protein expression with peripheral blood lymphocytes cell counts. The results are explicated as mean \pm standard deviation (SD). r, represent spearson correlation coefficients, $* \mathrm{P}<0.001$ : representative statistical significance difference vs Ctrl group.

Fig.3. Effects of CLCF1 on the JAK2/STAT3 pathway and RANKL/OPG ratio in Raji cells. (A) Western blot analysis of the effect of the rhCLCF1 on the activation of the JAK2/STAT3 pathway, with or without pretreatment by JAK2 inhibitor AG490. (B) Western blot analysis of the effect of the CLCF1 gene knockout on the JAK2/STAT3 pathway and RANKL and OPG. (C) Quantitative analyses of the relative protein levels. Data are representative of the average value of the intensity and the mean expression values were normalized to the actin. (D) Western blot analysis showing the effect of CLCF1 gene deletion on the RANKL/OPG ratio. *: representative statistical significance difference with Ctr group, $\mathrm{n}=3, \mathrm{n}$ is one independent culture experiments for Raji cells, Values are means $\pm \mathrm{SD}, *(\mathrm{P}<0.05), * *(\mathrm{P}<0.01)$ 
Fig. 4. Effect of CLCF1 gene knockout on osteogenic differentiation of the MG-63 cells in the Raji-MG-63 co-culture system. (A) Quantitative analyses of relative protein levels of the JAK2/STAT3 pathway and RANKL and OPG of MG-63 in the co-culture system. (B) Analyses of osteogenesis capacity as assessed by the ALP activity in MG-63 cells. (C) Quantitative analyses of relative osteogenesis capacity as shown by the alizarin red positive areas. Data are representative of the average value of the intensity. *: Representative statistical significance difference with the Ctr group, $n=3, n$ is one independent culture for Raji cells. Values are means $\pm \mathrm{SD}, * *(\mathrm{P}<0.01)$. 
Figures

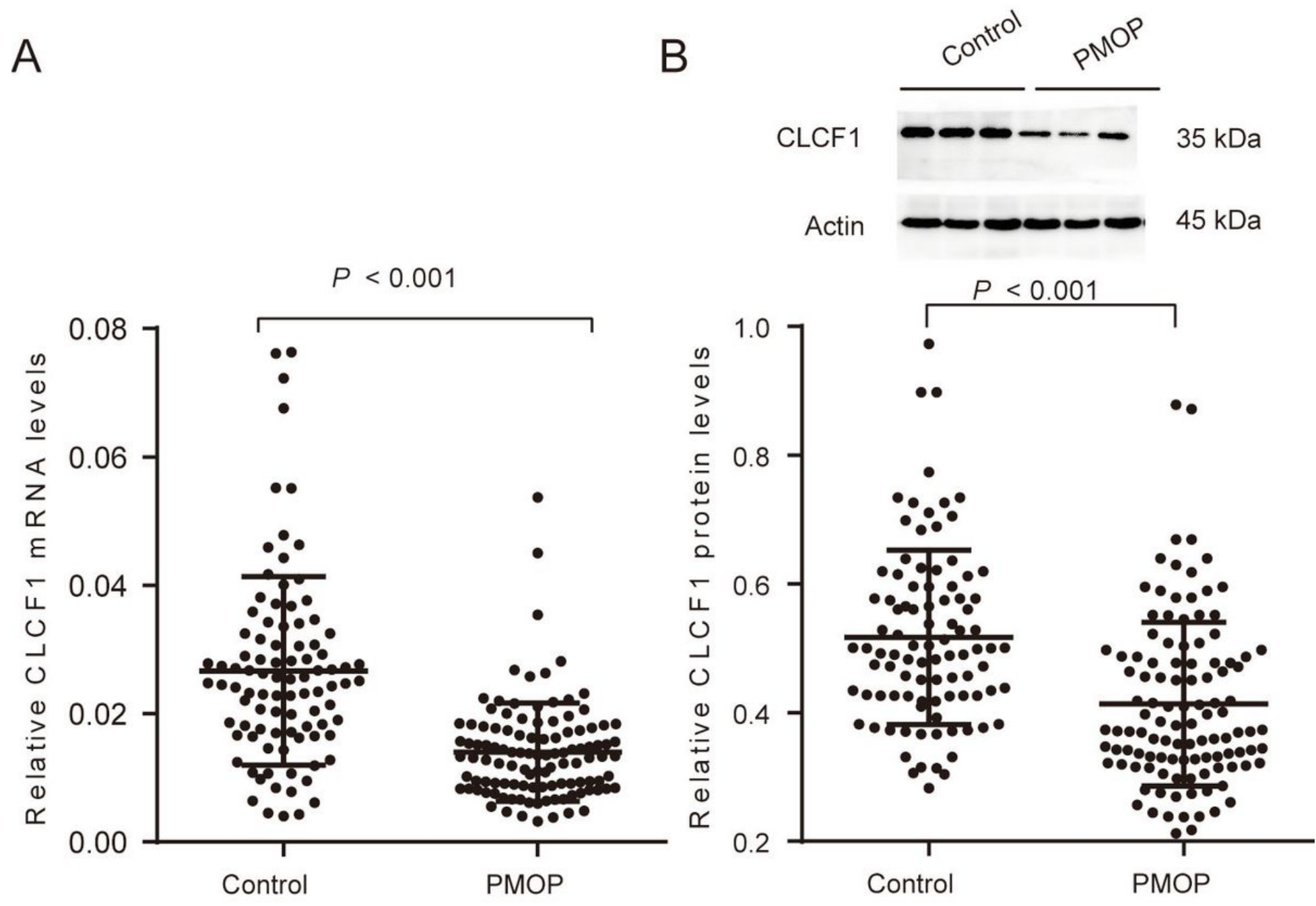

Figure 1

Altered expression of CLCF1 in postmenopausal osteoporosis patients. (A) The relative CLCF1 mRNA expression as detected by RT-qPCR. (B) Western blot analysis of the quantitative expression of the relative CLCF1 protein. The data represent the mean expression values normalized to the housekeeping gene Actin. Values are means \pm SD of three independent experiments, ${ }^{\star} \mathrm{P}<0.001$ compared to the $\mathrm{Ctrl}$ group. 


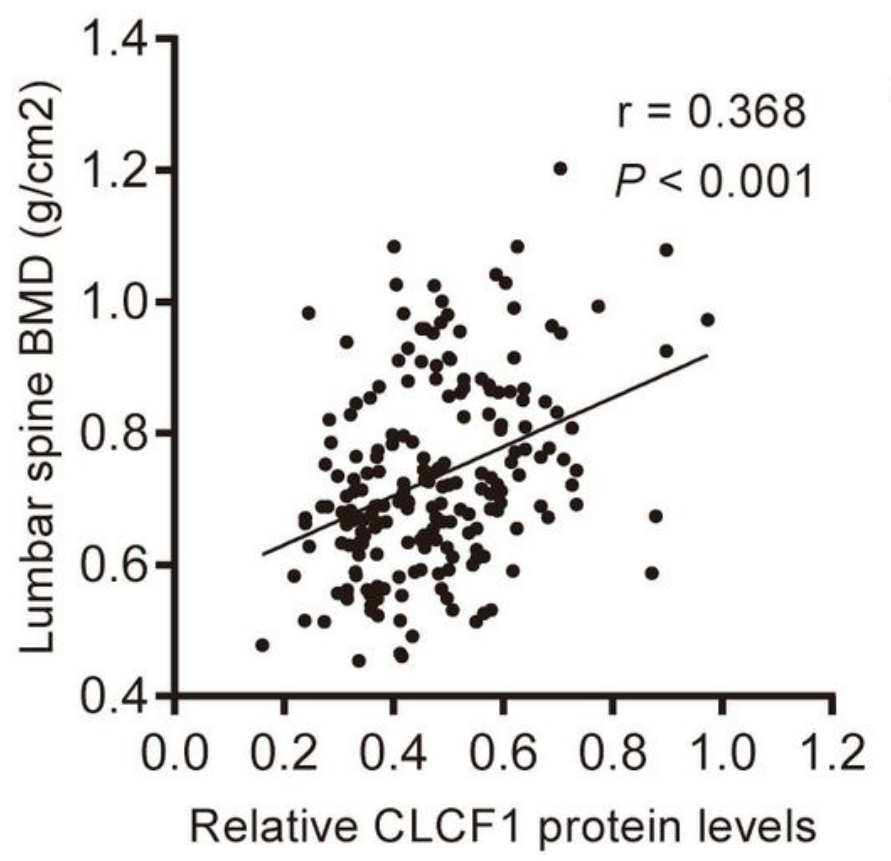

C

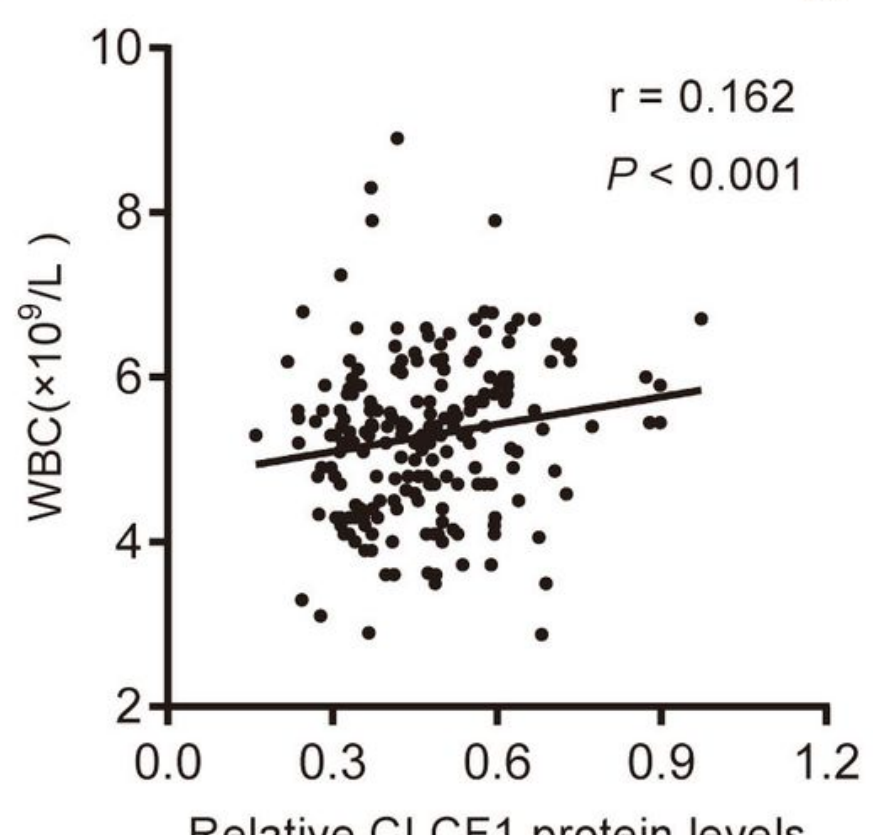

Relative CLCF1 protein levels
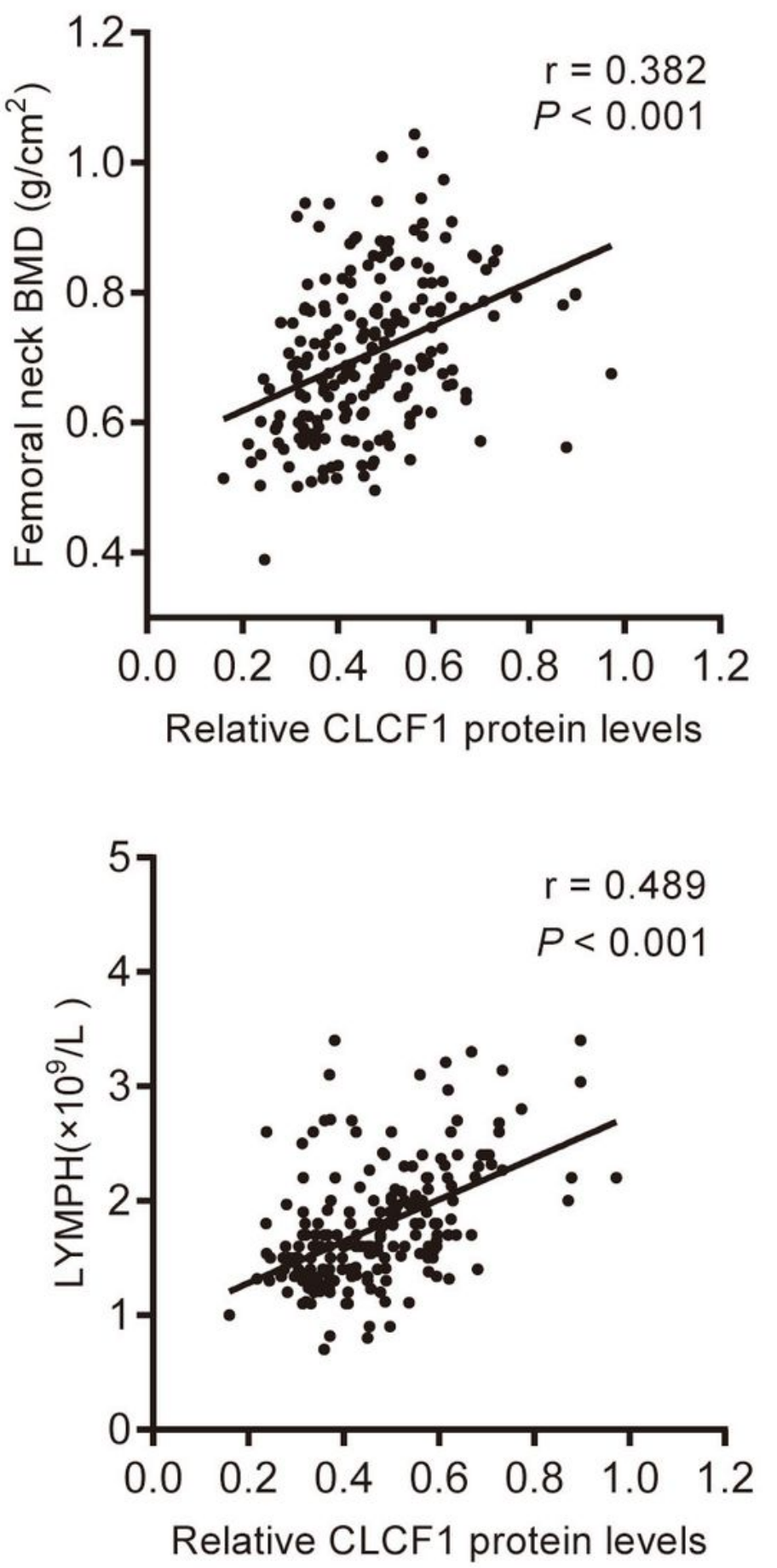

Figure 2

Association of CLCF1 expression profile with bone mineral density (BMD) and peripheral blood cell counts in postmenopausal osteoporosis patients. (A) The relationship between the CLCF1 protein expression in peripheral blood cells and the BMD of the lumbar spine. (B) The relationship between the CLCF1 protein expression and femoral neck BMD. (C) Association of CLCF1protein expression with peripheral blood white blood cell counts. (D) Association of CLCF1protein expression with peripheral 
blood lymphocytes cell counts. The results are explicated as mean \pm standard deviation (SD). $r$, represent spearson correlation coefficients, ${ }^{*} \mathrm{P}<0.001$ : representative statistical significance difference vs Ctrl group.

A

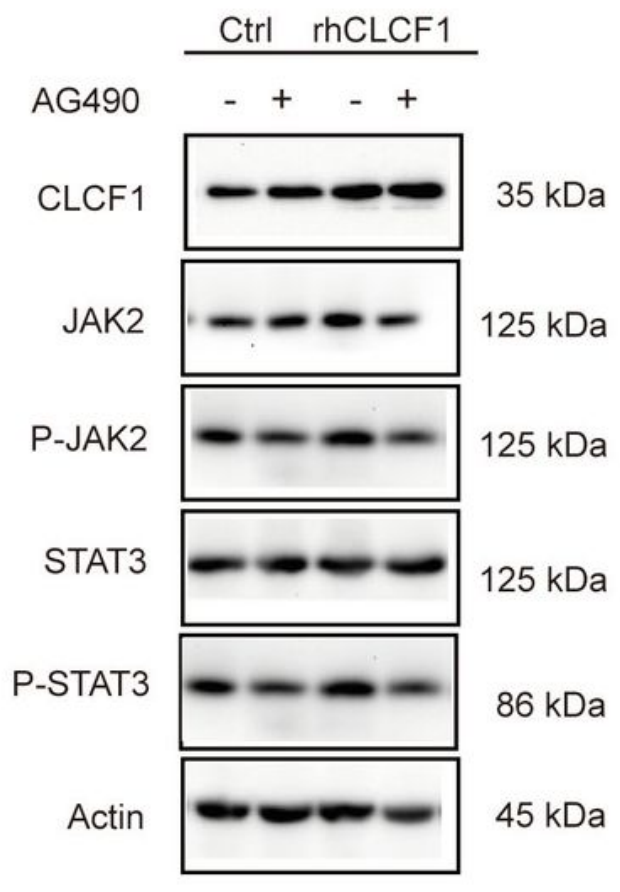

C

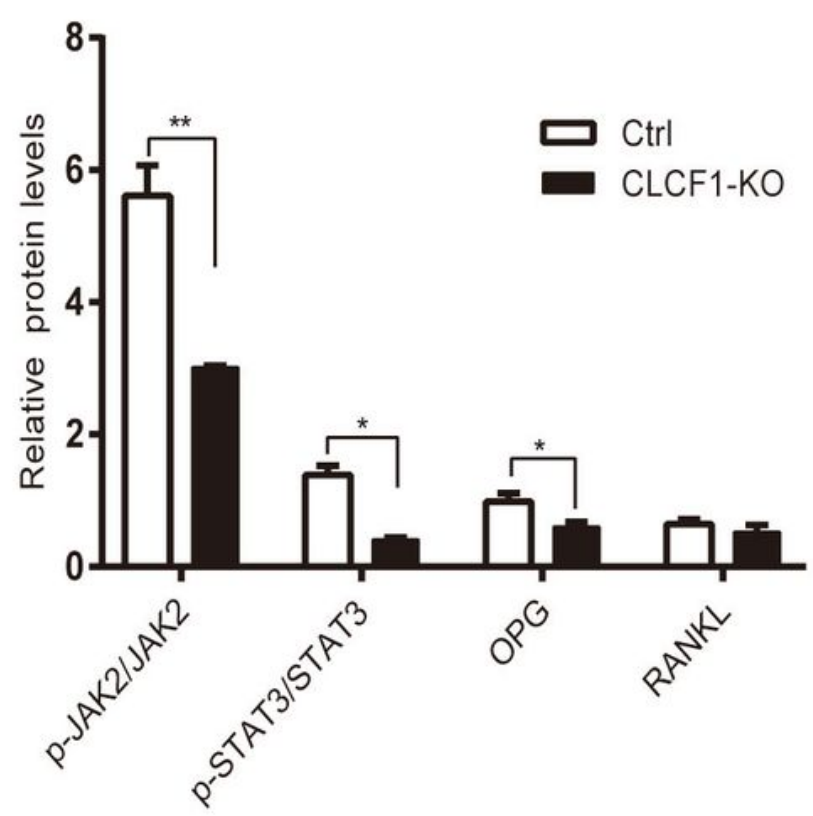

B

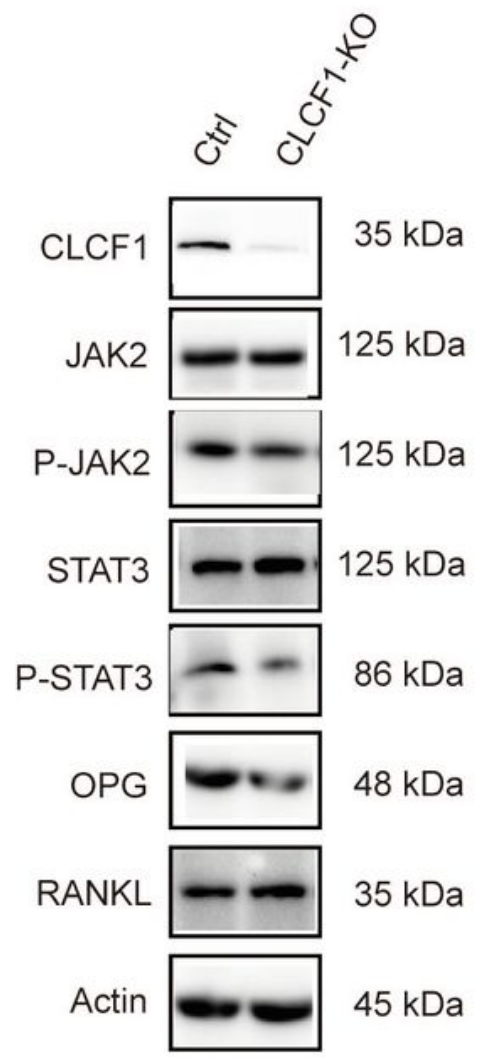

D

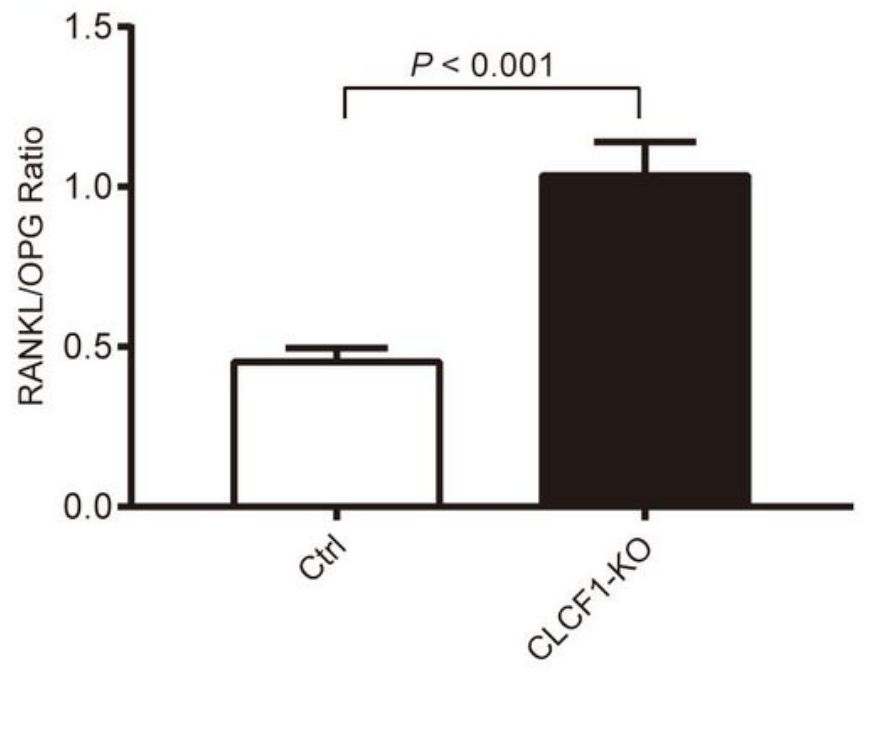

Figure 3

Effects of CLCF1 on the JAK2/STAT3 pathway and RANKL/OPG ratio in Raji cells. (A) Western blot analysis of the effect of the rhCLCF1 on the activation of the JAK2/STAT3 pathway, with or without 
pretreatment by JAK2 inhibitor AG490. (B) Western blot analysis of the effect of the CLCF1 gene knockout on the JAK2/STAT3 pathway and RANKL and OPG. (C) Quantitative analyses of the relative protein levels. Data are representative of the average value of the intensity and the mean expression values were normalized to the actin. (D) Western blot analysis showing the effect of CLCF1 gene deletion on the RANKL/OPG ratio. *: representative statistical significance difference with $C \operatorname{tr}$ group, $n=3, n$ is one independent culture experiments for Raji cells, Values are means $\pm S D, *(P<0.05)$, ** $(P<0.01)$.

A

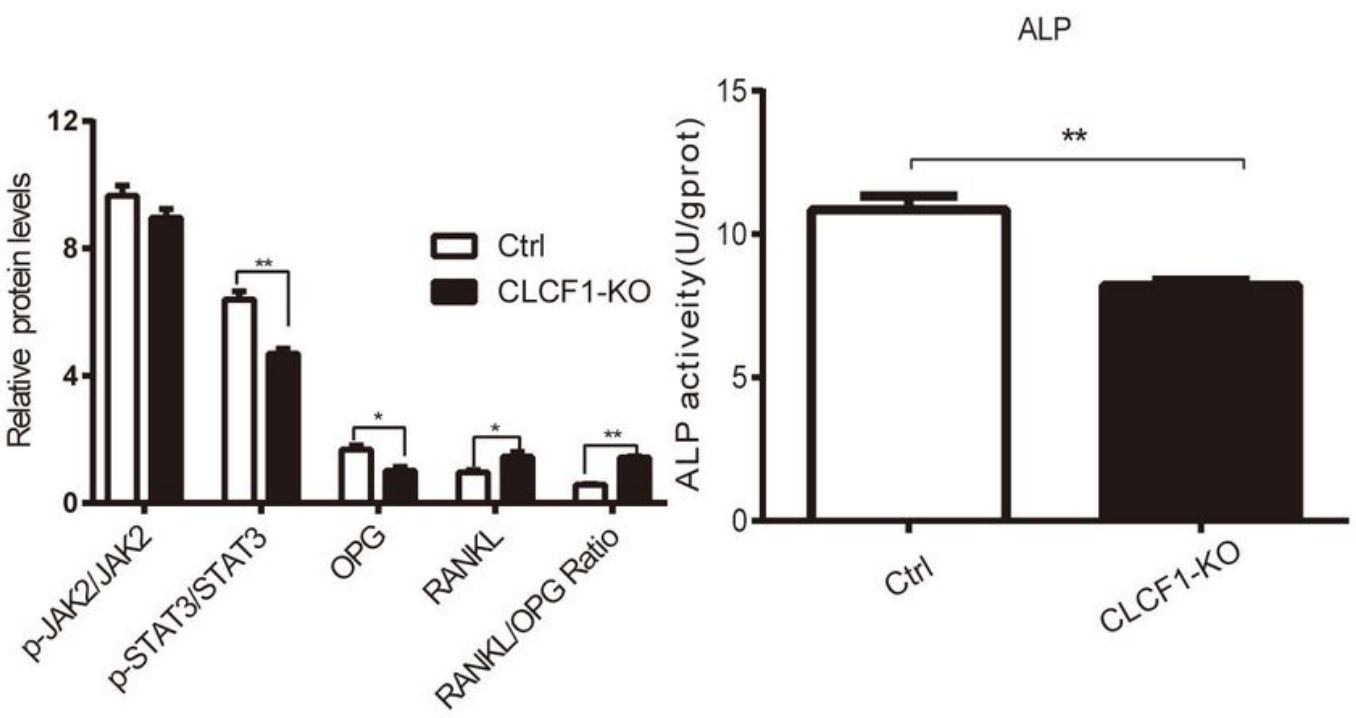

C Ctrl

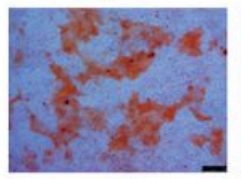

CLCF1-KO

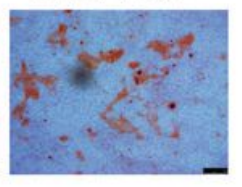

Alizarin Red

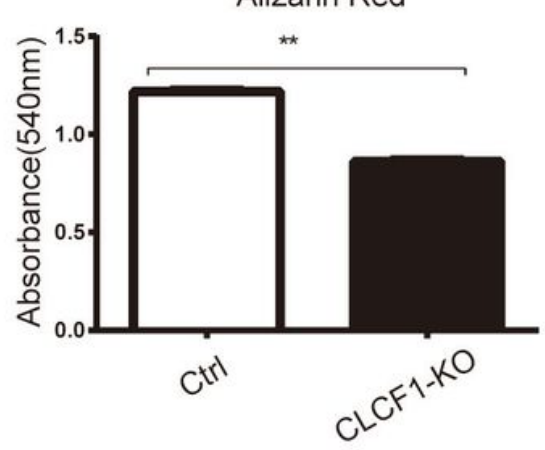

\section{Figure 4}

Effect of CLCF1 gene knockout on osteogenic differentiation of the MG-63 cells in the Raji-MG-63 coculture system. (A) Quantitative analyses of relative protein levels of the JAK2/STAT3 pathway and RANKL and OPG of MG-63 in the co-culture system. (B) Analyses of osteogenesis capacity as assessed by the ALP activity in MG-63 cells. (C) Quantitative analyses of relative osteogenesis capacity as shown by the alizarin red positive areas. Data are representative of the average value of the intensity. *:

Representative statistical significance difference with the Ctr group, $n=3, n$ is one independent culture for Raji cells. Values are means $\pm S D, * *(P<0.01)$.

\section{Supplementary Files}

This is a list of supplementary files associated with this preprint. Click to download.

- rawimagefilesofwesternblots.pdf 